\title{
Fault Diagnosis of IGBT Single-Phase Bridge Arm Based on Fuzzy Logic Genetic Algorithm
}

\author{
Jie WU
}

\begin{abstract}
Aiming at the problem that single-phase rectifier transformation is prone to failure in the use of three-phase rectifier transformation, this paper introduces fuzzy logic genetic algorithm into the fault detection process of IGBT single-phase bridge arm structure. The algorithm mainly analyzes the DC side current in detail in the frequency domain to judge the fault of IGBT open circuit. In the fault diagnosis of single-phase bridge arm structure, the system circuit model is analyzed by sensors by using fuzzy logic genetic algorithm to judge the type of fault. Finally, the experimental results show that this method can detect faults in a fixed sampling period and in the frequency domain. It can effectively eliminate the interference of other frequency signals and increase the reliability of the system and the accuracy of fault diagnosis.
\end{abstract}

Keywords: discrete fourier transform; igbt fault detection; open circuit fault; Single-phase pwm rectification

\section{INTRODUCTION}

In the test tooling for the aging of the power supply for rail transit air conditioning, taking the energy loss into consideration, it is necessary to use an energy-fed alternating current (AC) electronic load to carry out the aging test on the power supply for air conditioning [1-2]. In the selection of the pre-load simulation circuit of the general-fed AC electronic load [3-4], due to the excellent current control strategy of the single-phase PWM rectifier circuit, it can be used to simulate a number of different load features. Hence, it has been extensively used [5-8]. The single-phase PWM rectifier is mainly composed of two key components: IGBT and DC side capacitor. IGBT faults include short-circuit faults and open-circuit faults [9-10]. IGBT short circuit will induce over-current, and the fault can be diagnosed quickly based on over-current. IGBT open circuit will not lead to an apparent phenomenon, such as substantial over-current, over-voltage, and undervoltage. Hence, it is relatively difficult to diagnose and protect [11-13]. The disadvantage of the fault determination method based on the current is that the fault judgment time depends on the change of the current. If there is no drastic change of the current in the characteristic features of the fault, the response to the fault will be slow [14-15]. Although the fault determination method based on the voltage can be used to obtain a relatively fast fault response, it generally requires the use of a voltage sensor, which will increase the cost of the system. If the voltage sensor fails, the determination method will be useless. This can add risky factors to the reliability of the system instead.

In this paper, a fault detection method for the IGBT single-phase bridge arm structure based on the fuzzy logic genetic algorithm is put forward. The current is analyzed in the frequency domain. The type of faults is determined according to the specific spectrum component, which has enhanced the correctness of the fault judgment. At the same time, no additional sensors are added, which can avoid introducing any insecure factors.

\section{THE WORKING PRINCIPLE OF IGBT}

The IGBT is controlled by three poles: the gate $(\mathrm{G})$, the emitter (E) and the collector $(\mathrm{C})$. As shown in Fig. 1, the switching function of the IGBT is to form a channel by adding a positive gate voltage to provide a base current to the PNP transistor to turn on the IGBT. On the contrary, the reverse gate voltage is added to eliminate the channel, cut off the base current, and turn off the IGBT. It can be seen from Fig. 1 that if a positive driving voltage is applied between the gate and emitter of the IGBT, the MOSFET is turned on, so that the collector and base of the PNP transistor are in a low resistance state and the transistor is turned on. If the voltage between the gate and the emitter of the PNP is $0 \mathrm{~V}$, the MOSFET is turned off, cutting off the supply of the base current of the PNP transistor, so that the transistor is turned off.

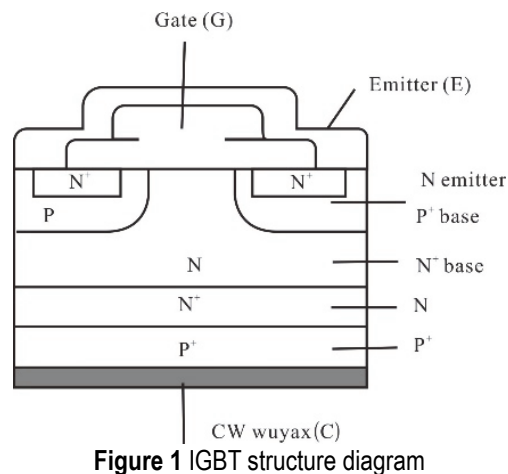

\section{FAULT DETECTION METHOD OF IGBT SINGLE-PHASE BRIDGE ARM STRUCTURE}

\subsection{Analysis of IGBT Open Circuit Fault Status}

Fig. 2 shows the topology of the single-phase PWM rectification, in which $U_{n}$ and $I_{n}$ stand for the voltage and current on the $\mathrm{AC}$ side of the circuit, respectively; $R_{n}$ stands for the line inductance, $L_{n}$ stands for the AC side current, $U_{d c}$ stands for the DC side voltage, and $C_{2}$ stands for the DC side energy storage capacitor. The singlephase PWM rectifier circuit mainly converts AC energy into DC energy through the switching state of the IGBT. Through the adjustment of the duration of different switching states, the DC side voltage can be controlled. At the same time, it can ensure that the input voltage and input current on the $\mathrm{AC}$ side are in the same phase, thereby 
guaranteeing the energy transmission of the unit power factor.

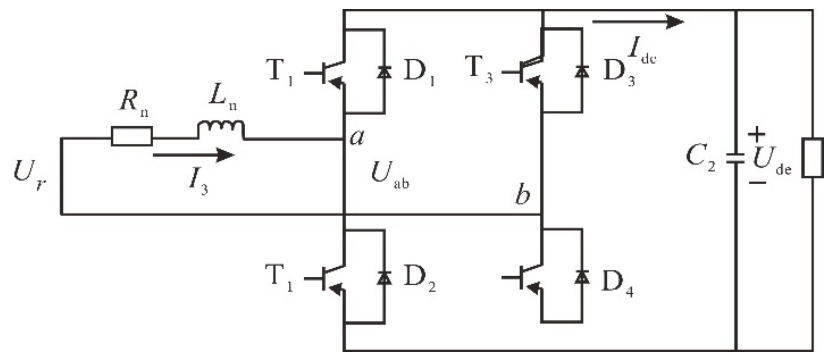

Figure 2 Schematic diagram of the single-phase PWM rectifier circuit

The switch function is defined as the following:

$$
\begin{aligned}
& S_{A}= \begin{cases}1 & T_{1} \text { or } D_{1} \text { on } \\
0 & T_{2} \text { or } D_{2} \text { on }\end{cases} \\
& S_{B}= \begin{cases}1 & T_{3} \text { or } D_{3} \text { on } \\
0 & T_{4} \text { or } D_{4} \text { on }\end{cases}
\end{aligned}
$$

In order to analyze the impact of the open circuit of the IGBT on the circuit, we assume that the direction of $I_{n}$ in Fig. 3 is the forward direction of the current. Firstly, the open circuit fault of T1 is analyzed (as shown in Fig. 3 below). When the current of $I_{n}$ is in the positive direction, an open circuit fault occurs in the T1. In order to ensure the current continuity of $L_{n}$, the current In can only flow to the DC side through D1. From the above analysis, it can be known that when the current $I_{n}$ is positive polarity, it will not affect $I_{n}$ if an open circuit fault occurs in the T1. However, when the polarity of the current $I_{n}$ is changed into negative, the current $I_{n}$ can no longer continue to flow through D1, which will inevitably have an impact on $I_{n}$. Combined with the switching commands of the singlephase PWM, the following three kinds of switching commands can be determined: (1010), (1001), and (1000), which comply with the above analysis.

Firstly, it is assumed that the current switch command of the circuit is (1010). When T1 is in a normal state, the current In passes through the bypass diodes D3 and T1, as shown in Fig. 3 below.

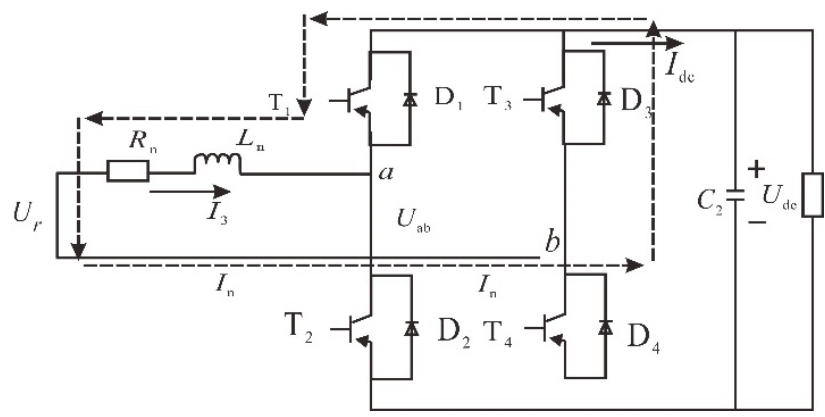

Figure $3 I_{n}$ in the normal state with the switch command of 1010
In this state, the alternating current (AC) side voltage will store energy in $L_{n}$, and $I_{n}$ is increased accordingly; at the same time, the energy stored in C2 is released to the load, and $U_{d c}$ is decreased.

\subsection{Detection of IGBT Open Circuit Fault Based on Iterative DFT}

Through the above analysis, it can be known that if the IGBT has an open circuit fault, it will inevitably change the waveform of In and convert it to the frequency domain, which can be clearly detected based on the size of the spectral component. According to the discrete Fourier transform [5], it can be known that the discretization of data in the frequency domain can be carried out to obtain the fuzzy logic genetic algorithm as the following:

$$
X_{\Delta f}=X_{n}=\sum_{n=0}^{N-1} X(n \Delta t) e^{(-f 2 \pi n k) / N}
$$

Eq. (1) is extended into a trigonometric function form as the following:

$$
X_{n}=\sum_{k=0}^{N-1} x(k \Delta t)\left[\cos \left(\frac{2 \pi n k}{N}\right)-j \sin \left(\frac{2 \pi n k}{N}\right)\right]
$$

Based on Eq. (2), it can be seen that $\cos \left(\frac{2 \pi n k}{N}\right)$ and $\sin \left(\frac{2 \pi n k}{N}\right)$ are the sampling points of the frequency component, and the sampling frequency is $f_{s}=\frac{1}{\Delta t}, T=N \Delta t$, in which $T$ stands for the length of the sampling time, and $N$ stands for the sampling point obtained by sampling at a sampling frequency of $f_{s}$ within the time of $T$. The values of the above two equations can be stored in the ROM of the DSP in the form of a sine table and a cosine table in advance, which can be taken out when it is necessary to carry out the calculation. From Eq. (2), it can be seen that the value of the frequency component can be divided into two parts for calculation as the following:

$$
\begin{aligned}
& X_{\text {cos }}(n)=\sum_{k=0}^{N-1} x(k \Delta t) \cos \left(\frac{2 \pi n k}{N}\right) \\
& X_{\text {sin }}(n)=\sum_{k=0}^{N-1} x(k \Delta t) \sin \left(\frac{2 \pi n k}{N}\right)
\end{aligned}
$$

Through analysis of Eq. (3) and Eq. (4), the following can be obtained, as shown in Tab. 1 .

\begin{tabular}{|c|l|c|}
\hline the $(n-N)$ th sampling & the $(n-(N+1))$ th sampling & the nth sampling \\
\hline the $(n-(N+1))$ th sampling & the $(n-(N+2))$ th sampling & the $(n+1)$ th sampling \\
\hline The $(n-(N+2))$ th sampling & the $(n-(N+3))$ th sampling & the $(n+2)$ th sampling \\
\hline
\end{tabular}


The last sampled value is replaced with the latest sampled value to ensure that there are always $N$ sample points during the calculation process, as shown in the following equation:

$$
\begin{aligned}
& X_{\cos }(n)=X_{\text {cos }}(n-1)+\operatorname{Sam}_{n}-\operatorname{Sam}_{n-N} \\
& X_{\sin }(n)=X_{\text {sin }}(n-1)+\operatorname{Sam}_{n}-\operatorname{Sam}_{n-N}
\end{aligned}
$$

In the above equations, $\operatorname{Sam}_{n}=x(k \Delta t) \cos \left(\frac{2 \pi n k}{N}\right)$, $\operatorname{Sam} C_{n-N}=x(k-N) \cos \left[\frac{2 \pi n(k-N)}{N}\right]$.

Through the analysis of the aforementioned iterative DFT algorithm process, it can be seen that for each sampled current, DFT calculation is carried out. It is necessary to perform only two subtractions, three additions, and two multiplications. Taking into consideration the presence of hardware multipliers in DSP, the time required for multiplication will not affect the real-time performance of fault detection. That is, the above algorithm can ensure real-time online detection of the IGBT open circuit fault.

\section{EXPERIMENT AND RESULT ANALYSIS}

For further verification of the correctness and effectiveness of the IGBT fault diagnosis method for the single-phase PWM rectifier based on the combination of the wavelet transform and the D-S evidence theory, as well as the diagnosis method for the DC side capacitor fault based on equivalent series resistance. Fig. 4 shows the experimental hardware platform established based on the main circuit diagram of the single-phase PWM rectifier. The related experiments are completed on this experimental platform, and the experimental platform parameters are consistent with the parameters of the simulation model.

The load power $P=3 \mathrm{~kW}$ is taken as an example. By blocking the IGBT pulse at a certain moment, the IGBT open circuit fault is simulated and set. Fig. 5 to Fig. 7 show the input current experimental waveform when the IGBT is normal, when it fails, and after it fails, respectively. The decision-making threshold value in the experiment is $\varepsilon=$
0.4 (which stands for a stricter identification of faults).

According to the experimental results of the IGBT failure in the single-phase PWM rectifier, by collecting a cycle of the input current data, combined with the IGBT fault diagnosis process in the single-phase PWM rectifier, the evidence fusion results of the IGBT failure in the single-phase PWM rectifier are shown in Tab. 2 as the following.

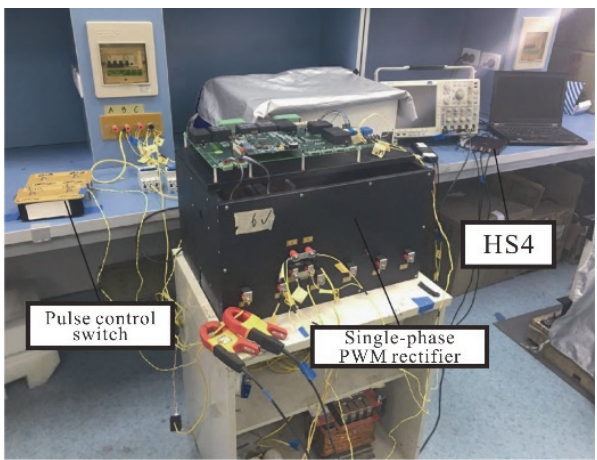

Figure 4 Physical diagram of the hardware platform in the experiment

From Tab. 2, it can be seen that when the IGBT is normal in the single-phase PWM rectifier, the results of the evidence fusion in the first and second half cycles are relatively close. Hence, it can be determined that no fault has occurred based on the diagnosis decision. Upon the failure of the IGBT in the single-phase PWM rectifier, the results of the evidence fusion in the first and second half cycles are significantly different, and the difference is greater than the threshold value of 0.4. Based on the diagnosis decision, it can be determined that there is an IGBT fault. Subsequently, the fault is further located based on the positive or negative mean values of the fault phase current.

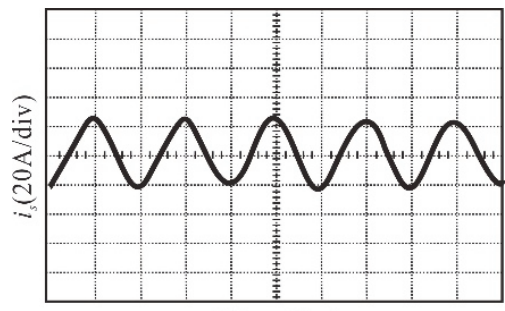

$t(10 \mathrm{~ms} / \mathrm{div})$

Figure 5 Input current waveform of the single-phase PWM rectifier when IGBT is normal

Table 2 Evidence fusion results of the single-phase PWM rectifier upon the failure of IGBT

\begin{tabular}{|c|c|c|c|}
\hline Upon the open circuit fault of the switch tube & Fault Features & First Half Cycle & Second Half Cycle \\
\hline \multirow{3}{*}{ No fault } & Wavelet energy degree & 0.4916 & 0.5084 \\
\cline { 2 - 4 } & Wavelet singularity degree & 0.5047 & 0.4953 \\
\cline { 2 - 4 } & Evidence fusion & 0.4964 & 0.5036 \\
\hline \multirow{3}{*}{ S1 } & Wavelet energy degree & 0.9695 & 0.0305 \\
& Wavelet singularity degree & 0.8584 & 0.1416 \\
\cline { 2 - 4 } & Evidence fusion & 0.9948 & 0.0052 \\
\cline { 2 - 4 } & Wavelet energy degree & 0.0438 & 0.9562 \\
\cline { 2 - 4 } & Wavelet singularity degree & 0.3013 & 0.6987 \\
\cline { 2 - 4 } & Evidence fusion & 0.0194 & 0.9806 \\
\hline \multirow{3}{*}{ S3 } & Wavelet energy degree & 0.0153 & 0.9847 \\
\cline { 2 - 4 } & Wavelet singularity degree & 0.1648 & 0.8352 \\
\cline { 2 - 4 } & Evidence fusion & 0.0031 & 0.9969 \\
\hline \multirow{3}{*}{ S4 } & Wavelet energy degree & 0.9703 & 0.0297 \\
\cline { 2 - 4 } & Wavelet singularity degree & 0.8463 & 0.1537 \\
\cline { 2 - 4 } & Evidence fusion & 0.9945 & 0.0055 \\
\hline
\end{tabular}

Based on the experimental results after the failure of

IGBT of the single-phase PWM rectifier, by collecting a 
cycle of the input current data, combined with the IGBT fault diagnosis process of the single-phase PWM rectifier, the evidence fusion results after the failure of IGBT of the single-phase PWM rectifier are shown in Tab. 3 as the following.

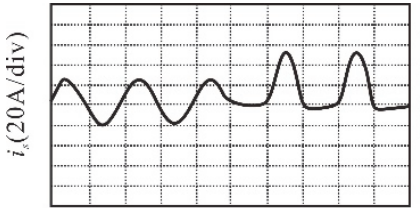

$t(10 \mathrm{~ms} / \mathrm{div})$

(a) After $\mathrm{S} 1$ tube failure

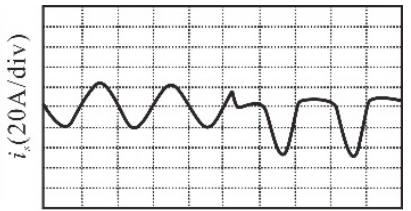

$t(10 \mathrm{~ms} / \mathrm{div})$

(b) After $\$ 2$ tube failure

Figure 6 Input current waveform of the single-phase PWM rectifier upon the failure of IGBT

Table 3 Evidence fusion results of the single-phase PWM rectifier after the failure of IGBT

\begin{tabular}{|c|c|c|c|}
\hline After the open circuit fault of the switch tube & Fault Features & First Half Cycle & Second Half Cycle \\
\hline \multirow{3}{*}{ S1 } & Wavelet energy degree & 0.9908 & 0.0092 \\
\hline & Wavelet singularity degree & 0.8384 & 0.1616 \\
\hline & Evidence fusion & 0.9982 & 0.0018 \\
\hline \multirow{3}{*}{ S2 } & Wavelet energy degree & 0.1233 & 0.8767 \\
\hline & Wavelet singularity degree & 0.4205 & 0.5795 \\
\hline & Evidence fusion & 0.0926 & 0.9074 \\
\hline \multirow{3}{*}{ S3 } & Wavelet energy degree & 0.0553 & 0.9447 \\
\hline & Wavelet singularity degree & 0.2751 & 0.7249 \\
\hline & Evidence fusion & 0.0217 & 0.9783 \\
\hline \multirow{3}{*}{ S4 } & Wavelet energy degree & 0.9890 & 0.0110 \\
\hline & Wavelet singularity degree & 0.8222 & 0.1778 \\
\hline & Evidence fusion & 0.9976 & 0.0024 \\
\hline
\end{tabular}

From Tab. 3, it can be seen that after the occurrence of IGBT failure of the single-phase PWM rectifier, the evidence fusion results in the first and second half cycles differ significantly, and the difference is greater than the threshold value 0.4. According to the diagnosis decision, it can be determined that there is an IGBT fault. Subsequently, based on the positive or negative mean value of the fault phase current, the fault is further located to the corresponding tube.

According to the IGBT fault diagnosis method of the single-phase PWM rectifier based on the combination of wavelet transform and D-S evidence theory put forward in this paper, the experimental study is completed by collecting the input current data. The results suggest that the IGBT health status determined by using the IGBT diagnosis method of the single-phase PWM rectifier under the experimental conditions is consistent with the simulation results, which has verified the correctness and effectiveness of the IGBT fault diagnosis method proposed under the simulation and experimental conditions.

Fast Fourier Transform (FFT) is used to perform spectrum analysis on the IGBT single-phase bridge arm current signal. The amplitude spectrum of the signal represents the energy of the corresponding frequency, and the phase spectrum represents the phase characteristic of the corresponding frequency. After the signal is Fourier transformed, different faults have a greater degree of discrimination in the amplitude and phase of the DC component, the third harmonic, and the fifth harmonic. Therefore, the amplitude and phase of the three components are the same as the positive half-cycle proportional coefficient. 21 features are used as the input of BP neural network (Back Propagation Neural Network, BPNN) for classification training. The activation function of the BP neural network is the Sigmoid function, which

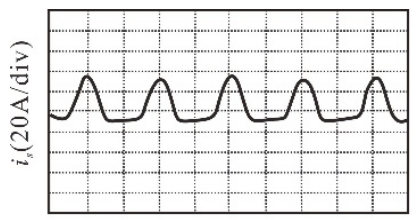

(a) $t(10 \mathrm{~ms} / \mathrm{div})$ failure of IGBT

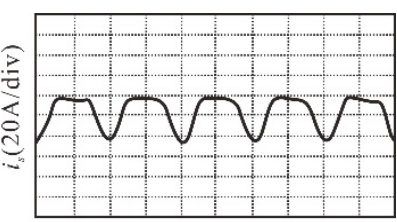
$t(10 \mathrm{~ms} / \mathrm{div})$
(b) After $\mathrm{S} 2$ tube failure
ase PWM rectifier after the initializes the weights and biases randomly; the feature vector is 21 dimensions, and the input layer of the BP neural network is 21 neurons; the hidden layer is a single layer with 20 neurons; the output layer is 73 Neurons. The fault diagnosis method based on Fourier transform and BP neural network (FFT-BPNN) has fault recognition accuracy rates of $94.4 \%$ and $89.4 \%$ in Case 1 and Case 2 test sets, respectively.

The DC component, the third harmonic and the fifth harmonic's amplitude, phase and positive half-cycle proportional coefficient extracted by Fourier transform are used as the input of SVM for classification learning. The fault diagnosis method based on Fourier transform and support vector machine (FFT-SVM) has a fault recognition accuracy rate of $95.3 \%$ and $89.6 \%$ in Case 1 and Case 2 test sets, respectively. A total of 15 features including the energy extracted by the three-layer wavelet and the positive half-cycle proportional coefficient are used as the input of the BP neural network. The activation function of the BP neural network is the Sigmoid function, which initializes the weights and biases randomly; the feature vector is 15 dimensions, and the input layer of the BP neural network is 15 neurons; the hidden layer is a single layer with 20 neurons; the output layer is 73 Neurons. The fault diagnosis method based on wavelet transform and BP neural network (WT-BPNN) has fault recognition accuracy rates of $95.8 \%$ and $9.9 \%$ in Case 1 and Case 2 test sets, respectively.

It can be seen from Fig. 8 and Fig. 9 that the accuracy of FFT-BPNN and FFT-SVM methods is low, and the accuracy of the FFT-BPNN and FFT-SVM methods fluctuates greatly in the presence of noise. The WT-BPNN method can effectively realize the diagnosis of the IGBT single-phase bridge arm fault. Fuzzy logic genetic algorithm (FLGA) has a diagnostic accuracy rate of up to $97.8 \%$, and it can still accurately identify faults under the 
condition of $10 \%$ Gaussian white noise.

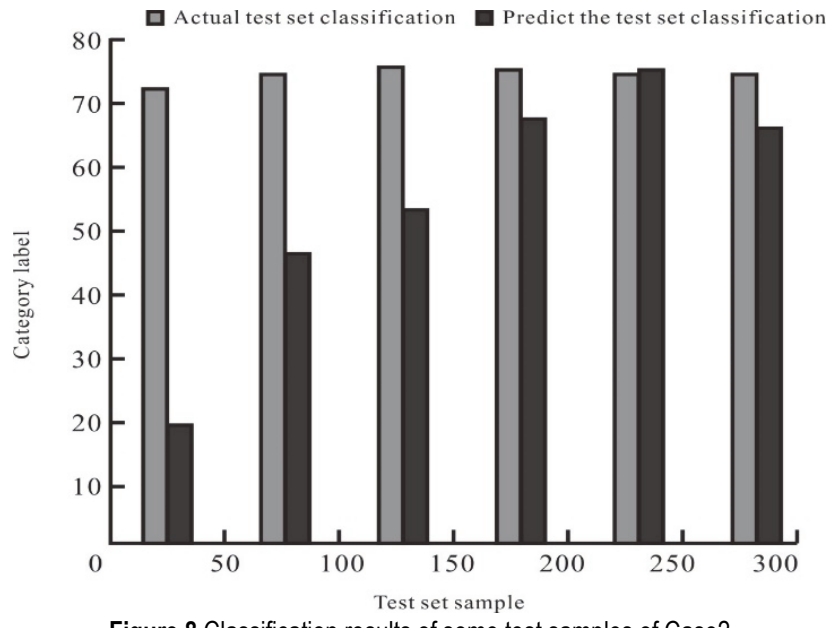

Figure $\mathbf{8}$ Classification results of some test samples of Case2

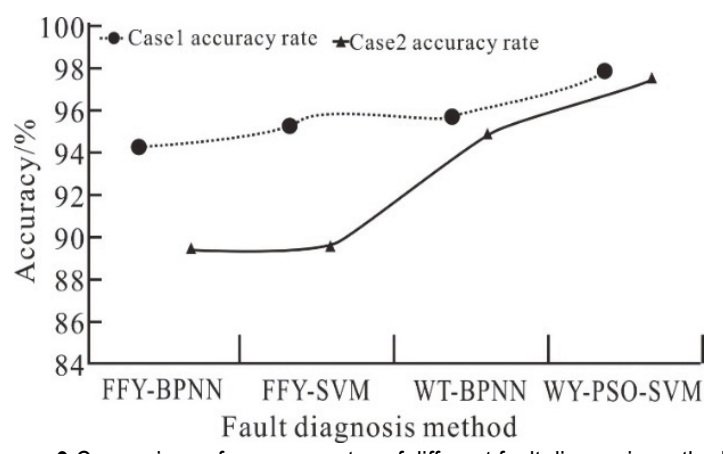

Figure 9 Comparison of accuracy rates of different fault diagnosis methods

\section{DISCUSSION}

According to the IGBT fault diagnosis method of the single-phase PWM rectifier based on the combination of wavelet transform and D-S evidence theory put forward in this paper, the experimental study is completed by collecting the input current data. The results suggest that the IGBT health status determined by using the IGBT diagnosis method of the single-phase PWM rectifier under the experimental conditions is consistent with the simulation results, which has verified the correctness and effectiveness of the IGBT fault diagnosis method proposed under the simulation and experimental conditions. Using the IGBT single-phase bridge arm current as the original current data sample, the comparative experiments of different diagnosis methods are carried out under the original current data sample and the data sample with $10 \%$ Gaussian white noise. The experimental results show that the fuzzy logic genetic algorithm fault diagnosis method can accurately identify the open circuit fault of IGBT single-phase bridge arm, compared with Fourier transform method and BP neural network algorithm. In the case of variable load and noise, it still has a high accuracy of $97.3 \%$.

\section{CONCLUSION}

In the proposed method, the problem is solved mainly by converting the fault detection issues in the time domain to the frequency domain. Through the detection of the harmonic content of the input current on the AC side, it can be determined whether an open circuit fault occurs.

The frequency domain analysis suggests that when an IGBT open circuit fault occurs, the frequency domain analysis of the input current will show that its harmonic content near the switching frequency will increase. Since the characteristic frequency analysis is carried out in the frequency domain, interference from the other frequency signals is eliminated. Hence, reliability is improved, and misjudgment can be avoided.

\section{Acknowledgement}

National Natural Science Foundation of China (Serial number of the project: 61271115); Education Department of Jilin Province (Serial number of the project: 201696).

\section{REFERENCES}

[1] Chen, Z., Chen, R., \& Chen, Z. (2013). A fault-tolerant parallel structure of single-phase full-bridge rectifiers for a wound-field doubly salient generator. IEEE Transactions on Industrial Electronics, 60(8), 2988-2996. https://doi.org/10.1109/TIE.2012.2203773

[2] Li, Z., Ma, H., Bai, Z., Wang, Y., \& Wang, B. (2017). Fast transistor open-circuit faults diagnosis in grid-tied threephase VSIs based on average bridge arm pole-to-pole voltages and error-adaptive thresholds. IEEE Transactions on Power Electronics, 33(9), 8040-8051. https://doi.org/10.1109/TPEL.2017.2773130

[3] Amir, A., Amir, A., Selvaraj, J., \& Rahim, N. A. (2020). Grid-connected photovoltaic system employing a singlephase t-type cascaded h-bridge inverter. Solar Energy, 199, 645-656. https://doi.org/10.1016/j.solener.2020.02.045

[4] Crouse, C. B., Hushmand, B., \& Martin, G. R. (1987). Dynamic soil-structure interaction of a single-span bridge. Earthquake Engineering \& Structural Dynamics, 15(6), 711729. https://doi.org/10.1002/eqe.4290150605

[5] Hua, Q., Li, Z., Zhang, B., Chen, W., Huang, X., \& Feng, Y. (2015). A rugged 650v SOI-based high-voltage half-bridge IGBT gate driver IC for motor drive applications. International Journal of Electronics, 102(5), 755-764. https://doi.org/10.1080/00207217.2014.938254

[6] Bhargavi, G., Rajasekharan, M. V., Costes, J. P., \& Tuchagues, J. P. (2013). A new end-on azido bridged $\mathrm{Mn}^{\mathrm{III}}$ single-chain magnet and its dimeric single molecule magnet polymorph. synthesis, structure and magnetic properties of $\left[\mathrm{Mn}(5 \text {-clsalpn }) \mathrm{N}_{3}\right]_{\mathrm{n}}$ and phenoxo bridged $\left[\mathrm{Mn}(5 \text {-clsalpn }) \mathrm{n}_{3}\right]_{2}$. Dalton Transactions, 42(22), 8113-8123. https://doi.org/10.1039/c3dt31966k

[7] Mayor, Á., Rizo, M., Monter, A. R., \& Bueno, E. J. (2019). Commutation behavior analysis of a dual 31-ANPC-VSC phase-leg PEBB using 4.5-kv and 1.5-ka HV-IGBT modules. IEEE Transactions on Power Electronics, 34(2), 1125-1141. https://doi.org/10.1109/TPEL.2018.2831602

[8] Xian, X., Qiang-Hua, H., Li-Yan, Y., Song-Qing, W., Yang, L., Hua, Y., Ai-Hua, Z., Xiao-Hong, M., Yu-Jie, P., \& Bing, C. (2017). Structure-based optimization of salt-bridge network across the complex interface of PTPN4 PDZ domain with its peptide ligands in neuroglioma. Computational biology and chemistry, 66, 63-68. https://doi.org/10.1016/j.compbiolchem.2016.11.005

[9] Li, C., Zhang, Y., Cao, Z., \& Dewei, X. U. (2017). Singlephase single-stage isolated ZCS current-fed full-bridge converter for high power $\mathrm{AC} / \mathrm{DC}$ applications. IEEE Transactions on Power Electronics, 32(9), 6800-6812. https://doi.org/10.1109/TPEL.2016.2623771

[10] Wang, C., Wang, K., Zheng, Z., Yang, B., Sun, K., \& Li, Y. (2019). Analysis and control of three-phase modular 
multilevel converters under the single arm fault condition. IEEE Transactions on Power Electronics, 34(9), 8293-8298. https://doi.org/10.1109/TPEL.2019.2906897

[11] Gurpinar, E. \& Castellazzi, A. (2015). Single-phase t-type inverter performance benchmark using si igbts, sic mosfets, and gan hemts. IEEE Transactions on Power Electronics, 31(10), 7148-7160. https://doi.org/10.1109/TPEL.2015.2506400

[12] Lamb, J. \& Mirafzal, B. (2017). Open-circuit IGBT fault detection and location isolation for cascaded multilevel converters. IEEE Transactions on Industrial Electronics, 64(6), 4846-4856. https://doi.org/10.1109/TIE.2017.2674629

[13] Ortiz, G., Uemura, H., Bortis, D., Kolar, J. W., \& Apeldoorn, O. (2013). Modeling of soft-switching losses of IGBTs in high-power high-efficiency dual-active-bridge DC/DC converters. IEEE Transactions on Electron Devices, 60(2), 587-597. https://doi.org/10.1109/TED.2012.2223215

[14] Wu, X., Xiong, C., Yang, S., Yang, H., \& Feng, X. (2020). A simplified space vector pulse width modulation scheme for three-phase cascaded h-bridge inverters. IEEE Transactions on Power Electronics, 35(4), 4192-4204. https://doi.org/10.1109/TPEL.2019.2934821

[15] Alavi, M., Luo, M., Wang, D., \& Bai, H. (2012). IGBT fault detection for three phase motor drives using neural networks. IEEE, 43(8), 1-8. https://doi.org/10.1109/ETFA.2012.6489593

\section{Contact information:}

\section{Jie WU}

1) College of electronic information engineering,

Changchun University of Science and Technology,

Changchun 130022, China

2) School of electrical and information engineering,

Beihua University,

Jilin 132021, China

E-mail: 2016200069@mails.cust.edu.cn 